\section{P-86 \\ PSYCHOSOCIAL WORK EXPOSURES OF THE JOB STRAIN MODEL AND ALL-CAUSE AND CAUSE-SPECIFIC MORTALITY: RESULTS FROM THE STRESSJEM PROJECT}

${ }^{1}$ Isabelle Niedhammer, Allison Milner, Thomas Coutrot, Béatrice Geoffroy-Perez, Anthony LaMontagne, Jean-François Chastang. 'Inserm DR GRAND OUEST, France

\subsection{6/OEM-2021-EPI.193}

Objectives Very little literature is available on the effects of psychosocial work exposures on mortality. The aim of the STRESSJEM project was to explore the prospective associations between these exposures and all-cause and cause-specific mortality.

Methods The STRESSJEM project was based on a French national representative sample of 798,547 male and 697,785 female employees for which data on job history on the 19762002 period were linked to mortality and causes of death data from the national registry. Job strain model exposures were imputed using a job-exposure matrix and three timevarying exposure measures were constructed: current, cumulative, and recency-weighted cumulative exposure. The prospective associations between these exposure measures and mortality were explored using Cox proportional hazards models.

Results 88,521 deaths occurred among men and 28,921 among women between 1976 and 2002. Low decision latitude, low social support, job strain, iso-strain, high strain, and passive job were found to be risk factors for all-cause mortality, cardiovascular mortality, suicide, and preventable mortality (including smoking- and alcohol-related mortality as well as external causes of death). The model with current exposure was the highest relative quality model. The fractions attributable to job strain were $5.64 \%$ and $4.13 \%$ for all-cause mortality, $5.64 \%$ and $6.44 \%$ for cardiovascular mortality, 5.29\% and $9.13 \%$ for suicide, and $5.1 \%$ and $3.1 \%$ for preventable mortality, among men and women respectively (though non-significantly different from zero for cardiovascular and preventable mortality among women).

Conclusion Our findings underlined the role of the job strain model exposures on all-cause and cause-specific mortality. The burden of mortality attributable to these exposures may be substantial, especially for suicide among women. Prevention oriented towards the psychosocial work environment may reduce mortality among working populations.

\section{P-87 UNEMPLOYMENT DURING PREGNANCY AND EXCESSIVE GESTATIONAL WEIGHT GAIN MEDIATED THE ASSOCIATION BETWEEN MATERNAL SHIFT WORK STATUS AND POSTPARTUM WEIGHT RETENTION}

${ }^{1}$ Chih-Fu Wei, Yue Leon Guo, Pau-Chung Chen, Mei-Huei Chen, Ching-Chun Lin, MengShan Tsai, Shio-Jean Lin, Wu-Shiun Hsieh, Jorge E Chavarro. ${ }^{1}$ Harvard School of Public Health, United States

\subsection{6/OEM-2021-EPI.194}

Introduction Shift work is associated with higher risk for mothers during pregnancy and increases future obesity risk for both mothers and children. However, only limited literature examined the effect of shift work on maternal body weight changes during pregnancy and after delivery.

Objectives To examine the association between maternal shift work and body weight changes during pregnancy periods, and to identify possible mediating factors.
Methods We extracted maternal weight and body mass index (BMI) during pregnancy and maternal shift work status from a population-based cohort study. The associations between maternal shift work and maternal weight retention at six months were evaluated with multivariable linear models, and we examined the mediation and interaction effect from excess gestational weight gain.

Results We included 13575 mothers giving birth to term singleton in the study population, and maternal shift work before pregnancy was associated with a 0.25 -kilogram higher postpartum weight retention at six months after adjusting for confounders (95\% CI: 0.11-0.40, $\mathrm{p}=0.001)$. Meanwhile, this association was mediated by excess gestational weight gain (Natural indirect effect: 0.12-kilogram increase, 95\% CI: $0.07-0.17$, p-value $<0$.001) and nonemployment during pregnancy (Natural indirect effect: 0.02-kilogram increase, 95\% CI: 0.00-0.03, p-value 0.006). Lastly, a 0.39-kilogram additive interaction (95\% CI: $0.12-0.65$, p-value 0.005 ) was identified for excessive gestational weight gain on the association between maternal shift work and postpartum weight gain at six months.

Conclusion Maternal shift work before pregnancy is associated with increased postpartum weight retention at six months, and this association was mediated by nonemployment status during pregnancy. Meanwhile, excessive gestational weight gain exacerbates the effect through additive interaction and mediation.

\section{P-90 MUSCULOSKELETAL DISORDERS BY GENDER AMONG WORKING SYRIAN REFUGEE CHILDREN IN LEBANON}

${ }^{1}$ Rima R Habib. 'American University of Beirut, Lebanon

\subsection{6/OEM-2021-EPI.195}

Background Due to the Syrian war, a massive number of Syrian refugees migrated to Lebanon, which forced children to engage in harmful jobs, mainly agriculture in the Bekaa Valley in the east of Lebanon. Children working in the agricultural sector experience ergonomic hazards associated with musculoskeletal disorders (MSDs).

Methods A survey of 4090 working Syrian refugee children between 8 and 18 years in the Bekaa Valley of Lebanon documented the variation in reporting MSDs between male and female working children. Data were collected on demographic, occupational, and socioeconomic indicators and musculoskeletal disorders. Statistical analyses were done using Stata V.15.0. Means and standard deviations were generated for continuous data, and frequencies and percentages for categorical data. Chi Square and independent t-test were used to test significant differences between males and females.

Results Around 4.4\% of the child workers experienced MSDs. The highest prevalence of MSDs was among children working in agriculture (73.2\%), with a higher prevalence among females (84.5\%) than males (63.2\%). The most common MSDs reported were back pain, feet pain, joint pain, and knee pain. Significantly, more females $(1.3 \%)$ reported wrist or hand pain than males $(0.6 \%)$.

Discussion The study showed that females and males were differently burdened with MSDs. Females child workers bore a higher burden than males due to working both inside and outside the house. Interventions and policies protecting children from harmful exposure and working conditions are 